\title{
The mixed quantum Rabi model
}

\begin{abstract}
Liwei Duan ${ }^{1}$, You-Fei Xie ${ }^{1}$ \& Qing-Hu Chen ${ }^{1,2 *}$
The analytical exact solutions to the mixed quantum Rabi model (ORM) including both one- and two-photon terms are found by using Bogoliubov operators. Transcendental functions in terms of $4 \times 4$ determinants responsible for the exact solutions are derived. These so-called $G$-functions with pole structures can be reduced to the previous ones in the unmixed QRMs. The zeros of $G$-functions reproduce completely the regular spectra. The exceptional eigenvalues can also be obtained by another transcendental function. From the pole structure, we can derive two energy limits when the twophoton coupling strength tends to the collapse point. All energy levels only collapse to the lower one, which diverges negatively. The level crossings in the unmixed QRMs are relaxed to avoided crossings in the present mixed QRM due to absence of parity symmetry. In the weak two-photon coupling regime, the mixed QRM is equivalent to an one-photon QRM with an effective positive bias, suppressed photon frequency and enhanced one-photon coupling, which may pave a highly efficient and economic way to access the deep-strong one-photon coupling regime.
\end{abstract}

The quantum Rabi model (QRM) describes the simplest and at the same time most important interaction between a two-level system (or qubit) and a single-mode bosonic cavity which is linear in the quadrature operators ${ }^{1}$. This model is a paradigmatic one in quantum optics for a long time. It has been reactivated in the past decade, due to the progress in many solid-state devices, such as the circuit quantum electrodynamics (QED) $)^{2,3}$, trapped ions ${ }^{4,5}$, and quantum dots 6 , where the strong coupling even ultra-strong coupling has been realized. Here we study a natural generalization of the QRM which exhibits both linear and non-linear couplings between the qubit and the cavity, i.e. the mixed QRM having both one- and two-photon terms, with Hamiltonian

$$
H=-\frac{\Delta}{2} \sigma_{x}+\omega a^{\dagger} a+\sigma_{z}\left(g_{1}\left(a^{\dagger}+a\right)+g_{2}\left[\left(a^{\dagger}\right)^{2}+a^{2}\right]\right),
$$

where $\Delta$ is the tunneling amplitude of the qubit, $\omega$ is the frequency of cavity, $\sigma_{x, z}$ are Pauli matrices describing the two-level system, $a\left(a^{\dagger}\right)$ is the annihilation (creation) bosonic operator of the cavity mode, and $g_{1}\left(g_{2}\right)$ is the linear (nonlinear) qubit-cavity coupling constant.

The nonlinear coupling appears naturally as an effective model for a three-level system when the third (off-resonant) state can be eliminated. The two-photon model has been proposed to apply to certain Rydberg atoms in superconducting microwave cavities ${ }^{7,8}$. Recently, a realistic implementation of the two-photon QRM using trapped ions has been proposed ${ }^{9}$. In the trapped ions, the atom-cavity coupling could be tuned to the collapse regime.

The mixed QRM described by Eq. (1) can also be implemented in the proposal of the circuit QED ${ }^{10}$ if non-zero DC current biases are applied. Using alternative methods, both linear and nonlinear interaction terms can be present in different circuit QED setup by Bertet et al. ${ }^{11,12}$. Besides the one-photon process, the two-photon process was also detected in the superconducting qubit and oscillator coupling system ${ }^{13}$. It was shown recently that a general Hamiltonian realized in the microwave driven ions can be used to simulate the QRM with nonlinear coupling ${ }^{14}$ by chosing properly the time dependent phase and in a suitable interaction picture. The combined linear and non-linear couplings can also be attained. More recently, Pedernales et al. proposed that a background of a $(1+1)$-dimensional black hole requires a QRM with both one- and two-photon terms that can be implemented in a trapped ion for the quantum simulation of Dirac particles in curved spacetime ${ }^{15}$. So the QRM with both one- and two-photon couplings is not only a generic model in the circuit QED and trapped ions, but also has applications in other realm of physics.

The unmixed QRMs, where either linear or nonlinear coupling is present, have been extensively studied for a few decades (for a review, please refer to refs. ${ }^{16-18}$ ). The solution based on the well-defined $G$-function with pole structures was only found for one-photon model by Braak ${ }^{19}$ in the Bargmann representation and two-photon model by Chen et al. ${ }^{20}$ using Bogoliubov transformations. These solutions have stimulated extensive

${ }^{1}$ Department of Physics and Zhejiang Province Key Laboratory of Quantum Technology and Device, Zhejiang University, Hangzhou, 310027, China. ${ }^{2}$ Collaborative Innovation Center of Advanced Microstructures, Nanjing University, Nanjing, 210093, China. *email: qhchen@zju.edu.cn 
research interests in the exact solutions to the unmixed QRMs and their variants with either one-photon ${ }^{21-26}$ or two-photon term ${ }^{27-34}$. In the literature, many analytical approximate but still very accurate results have also been given $^{35-47}$. In some limits of model parameters, the dynamics and quantum criticality have been studied exactly as well ${ }^{48-50}$. In the unmixed QRMs, the parity symmetry is very crucial to get the analytical solution in the closed system. Recently, the role of the parity symmetry has been characterized in the excitation-relaxation dynamics of the system as a function of light-matter coupling in the open systems ${ }^{51}$.

In the mixed QRM with both linear and nonlinear couplings, the parity symmetry is however broken naturally, and the analytical solution thus becomes more difficult ${ }^{52}$, compared to the unmixed models. In this paper, we propose an analytical exact solutions to this mixed QRM. We derive a G-function by the Bogoliubov transformations, which can be reduced to the previous G-functions for both one- and two-photon QRMs if either of the couplings appears. We demonstrate that the derived $G$-function can really yield the regular spectra by checking with the numerics. The exceptional eigenvalues are also given with the help of the non-degeneracy property in this mixed model due to the absence of any symmetry. Two kinds of formulae for the collapse points are derived. The avoided crossings are confirmed. The level collapse in the strong two-photon coupling regime is also discussed. Finally, we study the influence of mixed coupling by constructing an equivalent one-photon QRM with an effective positive bias where the photon frequency is suppressed and the one-photon coupling is enhanced.

\section{Solutions within the Bogoliubov Operators Approach}

In the basis of spin-up and spin-down states, the Hamiltonian (1) can be transformed to the following matrix form in units of $\omega=1$

$$
H=\left(\begin{array}{ll}
a^{\dagger} a+g_{1}\left(a^{\dagger}+a\right)+g_{2}\left[\left(a^{\dagger}\right)^{2}+a^{2}\right] & -\frac{\Delta}{2} \\
-\frac{\Delta}{2} & a^{\dagger} a-g_{1}\left(a^{\dagger}+a\right)-g_{2}\left[\left(a^{\dagger}\right)^{2}+a^{2}\right]
\end{array}\right) .
$$

First, we perform Bogoliubov transformation

$$
\begin{aligned}
& A=S(r) D^{\dagger}(w) a D(w) S^{\dagger}(r)=u a+v a^{\dagger}+w, \\
& A^{\dagger}=S(r) D^{\dagger}(w) a^{\dagger} D(w) S^{\dagger}(r)=u a^{\dagger}+v a+w,
\end{aligned}
$$

to generate a new bosonic operator, where $S(r)$ is the squeezing operator and $D(w)$ is the displaced operator

$$
S(r)=e^{\frac{r}{2}\left(a^{2}-a^{\dagger 2}\right)}, D(w)=e^{w\left(a^{\dagger}-a\right)},
$$

with $r=\operatorname{arc} \cosh u$. If set

$$
u=\sqrt{\frac{1+\beta}{2 \beta}}, v=\sqrt{\frac{1-\beta}{2 \beta}}, w=\frac{u^{2}+v^{2}}{u+v} g_{1},
$$

with $\beta=\sqrt{1-4 g_{2}^{2}}$, we have a simple quadratic form of one diagonal Hamiltonian matrix element

$$
H_{11}=\frac{A^{\dagger} A-v^{2}-w^{2}}{u^{2}+v^{2}} .
$$

The eigenstates of $H_{11}$ are the number states $|n\rangle_{A}$ which can be written in terms of the Fock states $|n\rangle$ in original bosonic operator $a$ as

$$
|n\rangle_{A}=S(r) D^{\dagger}(w)|n\rangle
$$

Similarly, we can introduce another operator

$$
\begin{aligned}
& B=S^{\dagger}(r) D^{\dagger}\left(w^{\prime}\right) a D\left(w^{\prime}\right) S(r)=u a-v a^{\dagger}+w^{\prime}, \\
& B^{\dagger}=S^{\dagger}(r) D^{\dagger}\left(w^{\prime}\right) a^{\dagger} D\left(w^{\prime}\right) S(r)=u a^{\dagger}-v a+w^{\prime},
\end{aligned}
$$

with

$$
w^{\prime}=\frac{u^{2}+v^{2}}{v-u} g_{1}
$$

which yields a simple quadratic form of the other diagonal Hamiltonian matrix element

$$
H_{22}=\frac{B^{\dagger} B-v^{2}-w^{\prime 2}}{u^{2}+v^{2}} .
$$

Note that if $g_{2}=0$, we have $w=g_{1}$ and $w^{\prime}=-g_{1}$, which are exactly the same as those in the one-photon QRM ${ }^{20}$. Similarly, the eigenstates of $H_{22}$ are the number states $|n\rangle_{B}$ which can be written in terms of the Fock states $|n\rangle$ in original bosonic operator $a$ as 


$$
|n\rangle_{B}=S^{\dagger}(r) D^{\dagger}\left(w^{\prime}\right)|n\rangle .
$$

In terms of the Bogoliubov operator $A$, the Hamiltonian can be written as

$$
H=\left(\begin{array}{ll}
\frac{A^{\dagger} A-v^{2}-w^{2}}{u^{2}+v^{2}} & -\frac{\Delta}{2} \\
-\frac{\Delta}{2} & H^{\prime}{ }_{22}
\end{array}\right),
$$

where

$$
H^{\prime}{ }_{22}=\left(\left(u^{2}+v^{2}\right)+4 g_{2} u v\right) A^{\dagger} A-2 u v\left(\left(A^{\dagger}\right)^{2}+A^{2}\right)-2(u-v)^{2} w\left(A^{\dagger}+A\right)+h_{A},
$$

with

$$
h_{A}=v^{2}+(u-v)^{2} w^{2}\left(1-2 g_{2}\right)+2 g_{1}(u-v) w+2 g_{2} u v .
$$

In principle, the eigenfunctions of the Hamiltonian can be expanded in terms of the number states of operator $A$

$$
|\psi\rangle_{A}=\sum_{n=0}^{\infty} \sqrt{n !}\left(\begin{array}{c}
e_{n}|n\rangle_{A} \\
f_{n}|n\rangle_{A}
\end{array}\right) .
$$

Projecting the Schrödinger equation onto $|n\rangle_{A}$ gives

$$
\begin{gathered}
e_{n}=\frac{\Delta / 2}{\frac{n-v^{2}-w^{2}}{u^{2}+v^{2}}-E} f_{n}, \\
f_{n+2}=\frac{-\frac{\Delta}{2} e_{n}+\left[\Omega(n, E)+h_{A}\right] f_{n}-2(u-v)^{2} w\left(f_{n-1}+(n+1) f_{n+1}\right)-2 u v f_{n-2}}{2 u v(n+1)(n+2)},
\end{gathered}
$$

with

$$
\begin{aligned}
\Omega(n, E) & =\left(u^{2}+v^{2}+4 g_{2} u v\right) n-E \\
& =\frac{\left(1+4 g_{2}^{2}\right)}{\beta} n-E .
\end{aligned}
$$

This is actually a five terms recurrence relation for $f_{n}$. All coefficients $e_{n}$ and $f_{n}$ for $n>1$ are determined in terms of $f_{0}$ and $f_{1}$ linearly.

In terms of the Bogoliubov operator $B$, the Hamiltonian can be written as

$$
H=\left(\begin{array}{ll}
H^{\prime} & -\frac{\Delta}{2} \\
-\frac{\Delta}{2} & \frac{B^{\dagger} B-v^{2}-w^{\prime 2}}{u^{2}+v^{2}}
\end{array}\right),
$$

where

$$
H^{\prime}{ }_{11}=\left(\left(u^{2}+v^{2}\right)+4 g_{2} u v\right) B^{\dagger} B-2(u+v)^{2} w^{\prime}\left(B^{\dagger}+B\right)+2 u v\left(\left(B^{\dagger}\right)^{2}+B^{2}\right)+h_{B},
$$

with

$$
h_{B}=v^{2}+(u+v)^{2} w^{\prime 2}\left(1+2 g_{2}\right)-2 g_{1}(u+v) w^{\prime}+2 g_{2} u v .
$$

We can express the eigenfunctions as

$$
|\psi\rangle_{B}=\sum_{n=0}^{\infty} \sqrt{n !}\left(\begin{array}{c}
f^{\prime}{ }_{n}|n\rangle_{B} \\
e^{\prime}{ }_{n}|n\rangle_{B}
\end{array}\right) .
$$

Similarly, we can get

$$
e^{\prime}{ }_{n}=\frac{\frac{\Delta}{2}}{\frac{n-v^{2}-w^{\prime 2}}{u^{2}+v^{2}}-E} f^{\prime}{ }_{n},
$$


and the similar five terms recurrence relation for ${f^{\prime}}_{n}$. Analogously, all coefficients $e^{\prime}{ }_{n}$ and $f^{\prime}{ }_{n}$ for $n>1$ are determined through ${f^{\prime}}_{0}$ and ${f^{\prime}}_{1}$ linearly.

Except for the crossing points in the energy spectra, the eigenstates are nondegenerate. Two wavefunctions in terms of operators $A$ and $B$ correspond to the same eigenstate. Therefore, they should be proportional with each other by a constant $r$,

$$
\sum_{n=0}^{\infty} \sqrt{n !}\left(\begin{array}{c}
e_{n}|n\rangle_{A} \\
f_{n}|n\rangle_{A}
\end{array}\right)=r \sum_{n=0}^{\infty} \sqrt{n !}\left(\begin{array}{c}
f^{\prime}{ }_{n}|n\rangle_{B} \\
e^{\prime}{ }_{n}|n\rangle_{B}
\end{array}\right) .
$$

We will set $r=1$, because only ratios among $f_{0}, f_{1}, r f^{\prime}{ }_{0}$ and $r f^{\prime}{ }_{1}$ are relevant. In this case we can absorb $r$ into new ${f^{\prime}}_{0}$ and ${f^{\prime}}_{1}$. Then we have

$$
\begin{aligned}
& \sum_{n=0}^{\infty} \sqrt{n !} e_{n}|n\rangle_{A}=\sum_{n=0}^{\infty} \sqrt{n !} f_{n}^{\prime}|n\rangle_{B}, \\
& \sum_{n=0}^{\infty} \sqrt{n !} f_{n}|n\rangle_{A}=\sum_{n=0}^{\infty} \sqrt{n !} e^{\prime}{ }_{n}|n\rangle_{B} .
\end{aligned}
$$

In the unmixed QRM, the well-defined $G$-functions can be derived by using the lowest number state $|0\rangle$ in the original Fock basis for the one-photon model ${ }^{19,20}$, and two lowest number states $|0\rangle$ and $|1\rangle$ for the two-photon model $^{20,30}$. Here, we also project Eqs. (18) and (19) onto two original number states $|0\rangle$ and $|1\rangle$, and then obtain the following 4 equations

$$
\begin{aligned}
& G^{(0,0)}=\sum_{n=0}^{\infty} \sqrt{n !}\left[f_{n}\langle 0 \mid n\rangle_{A}-e^{\prime}{ }_{n}\langle 0 \mid n\rangle_{B}\right]=0, \\
& G^{(0,1)}=\sum_{n=0}^{\infty} \sqrt{n !}\left[f_{n}\langle 1 \mid n\rangle_{A}-e^{\prime}{ }_{n}\langle 1 \mid n\rangle_{B}\right]=0, \\
& G^{(1,0)}=\sum_{n=0}^{\infty} \sqrt{n !}\left[e_{n}\langle 0 \mid n\rangle_{A}-{f^{\prime}}_{n}\langle 0 \mid n\rangle_{B}\right]=0, \\
& G^{(1,1)}=\sum_{n=0}^{\infty} \sqrt{n !}\left[e_{n}\langle 1 \mid n\rangle_{A}-{f^{\prime}}_{n}\langle 1 \mid n\rangle_{B}\right]=0 .
\end{aligned}
$$

They form 4 sets of linear homogeneous equations with 4 unknown variables $f_{0}, f_{1}, f^{\prime}{ }_{0}$, and $f^{\prime}$. Nonzero solutions require the vanishing of the following $4 \times 4$ determinant

$$
G(E)=\left|G_{i, j}\right|=0,
$$

where elements $G_{i, j} s$ are just coefficients before $f_{0}, f_{1}, f^{\prime}$, and $f^{\prime}{ }_{1}$ in Eqs. (20-23).

Equation (24) is just the $G$-function of the present mixed QRM. Its zeros thus give all regular eigenvalues of the mixed QRM, which in turn give the eigenstates using Eq. (12) or Eq. (15). Note from the coefficients in Eqs. (13) and (14) that this $G$-function is a well-defined transcendental function. Thus analytical exact solutions have been formally found. In the next section, we will employ it to analyze the characteristics of the spectra.

Note that for the one-photon QRM $\left(g_{2}=0\right)$, the parity symmetry leads to

$$
e_{n}^{\prime}= \pm(-1)^{n} e_{n}, f_{n}^{\prime}= \pm(-1)^{n} f_{n} .
$$

then Eq. (20) becomes

$$
G^{(0,0)}(E)=\sum_{n=0}^{\infty} \sqrt{n !}\left[f_{n}\langle 0 \mid n\rangle_{A} \mp(-1)^{n} e_{n}\langle 0 \mid n\rangle_{B}\right],
$$

which is just the $G$-function of one-photon QRM $\left(g_{2}=0\right)^{19}$. Similarly, Eq. (20) (Eq. (21)) can be reduced to the previous ones ${ }^{20,30}$ of the two-photon QRM $\left(g_{1}=0\right)$ in the subspace with even (odd) bosonic number.

In various unmixed QRMs, such as one-photon ${ }^{19,20}$, two-photon ${ }^{30}$ or two-mode ${ }^{53} \mathrm{QRM}$, the coefficients of the eigenstates satisfy a three-term recurrence relation which can be achieved by performing the Bogoliubov transformation. All these $G$-functions with explicit pole structures have been summarized in Eq. (27) of ref. ${ }^{53}$. But in the mixed model, the Hilbert space cannot be separated into invariant subspaces due to the lack of parity symmetry, so the recurrence relation of the coefficients $\left\{f_{n}\right\}$ is of higher order, as seen in Eq. (14). A similar behavior also happens in the Dicke model ${ }^{5,55}$. The possible reason is that the symmetry does not suffice to label each state uniquely, indicating that mixed QRM is non-integrable according to Braak's criterion for quantum intergrability ${ }^{19}$. 
(a) $\Delta=0.5, g_{1}=0.1, g_{2}=0.2$
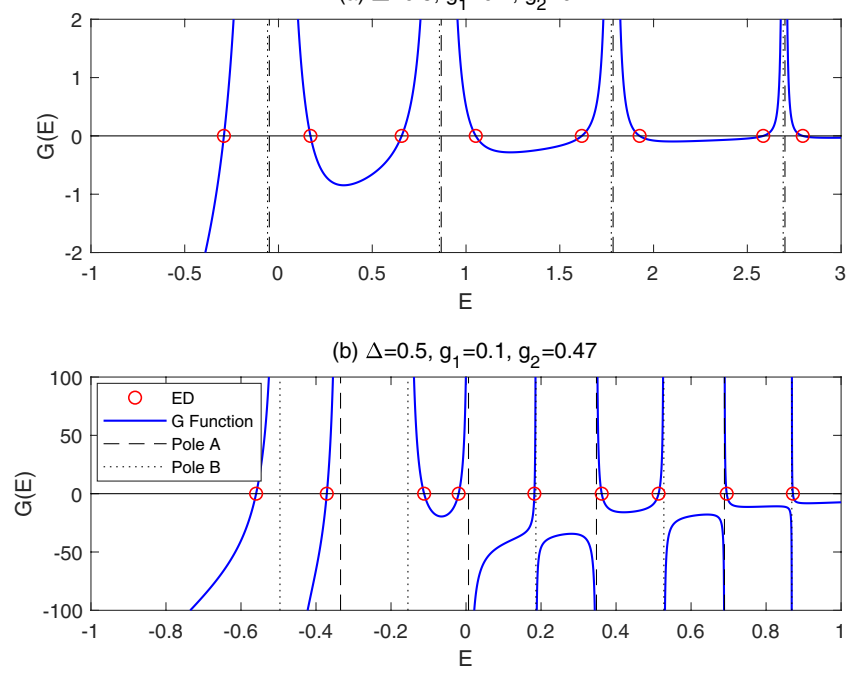

Figure 1. $G$-curves for $\Delta=0.5, g_{1}=0.1$. (a) $g_{2}=0.2$, (b) $g_{2}=0.47$. The blue solid lines denote the $G$-curves. The dash (dot) lines denote poles associated with $A(B)$ operator. The red circles denote the results calculated by numerical diagonalization.
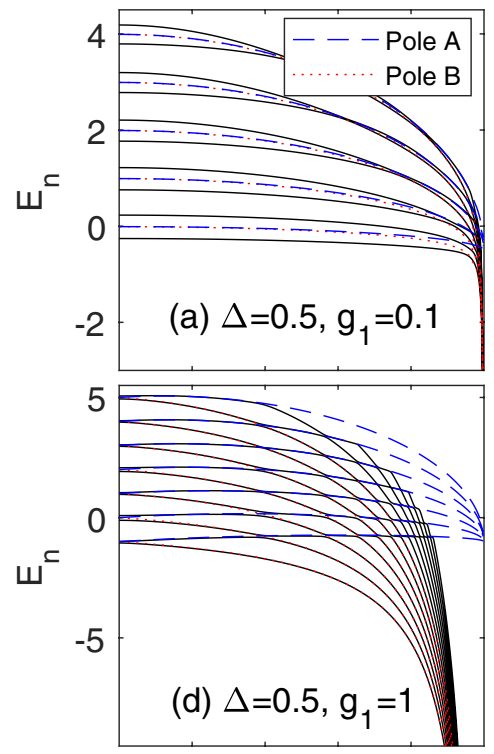

$\begin{array}{cccc}0.1 & 0.2 & 0.3 & 0.4 \\ g_{2} & \end{array}$
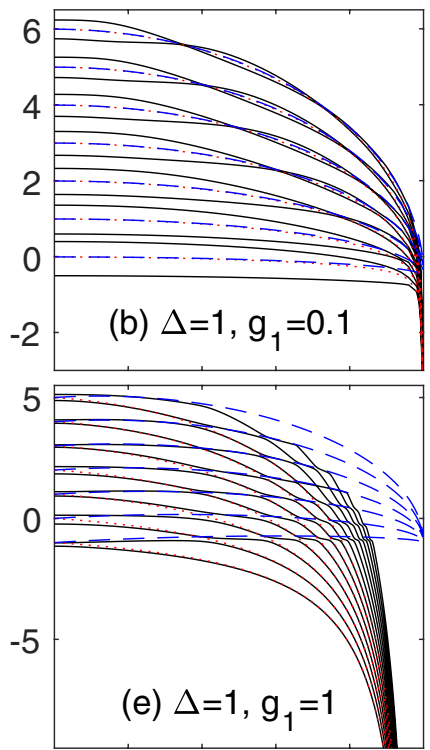

$\begin{array}{llll}0.1 & 0.2 & 0.3 & 0.4\end{array}$ $\mathrm{g}_{2}$
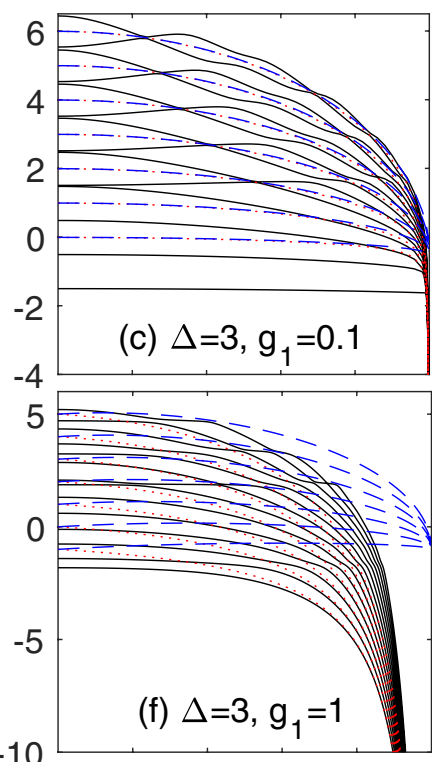

$\begin{array}{llll}0.1 & 0.2 & 0.3 & 0.4\end{array}$ $g_{2}$

Figure 2. Energy spectra as a function of $g_{2}$. The solid black lines denote the energy spectra obtained from the $G$-function. The blue dash (red dot) lines denote poles associated with $A(B)$ operator.

\section{Exact Spectra}

Regular spectra. To show the validity of the $G$-function (24), we first check with independent numerics. The $G$-curves as a function of $E$ for $\Delta=0.5, g_{1}=0.1, g_{2}=0.2$ and 0.47 are depicted in Fig. 1 . We find that the zeros of $G$-function indeed yield the true eigenvalues by comparing with the numerical diagonalization in truncated Hilbert spaces with sufficiently high dimension.

Then we plot the energy spectra calculated by the $G$-function (24) in Fig. 2. Checking with numerics, our $G$-function reproduces completely all eigenvalues of the present mixed model. When $g_{2}$ is close to $1 / 2$, the energy spectra collapse to negative infinity. The parity symmetry in this mixed QRM is lacking, so in principle, the energy degeneracy should be relieved, and level crossings should be absent. However, as shown in Fig. 2(a-c), it seems that some crossings still occur for the small $g_{1}$. It will be shown later that these "crossing" can be actually discerned as avoided crossings. 
(a) $\Delta=0.5, g_{1}=0.1$

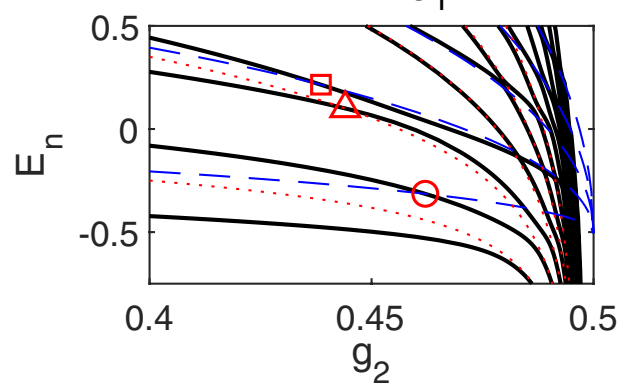

(c) $\Delta=0.5, g_{1}=0.1, m=0$

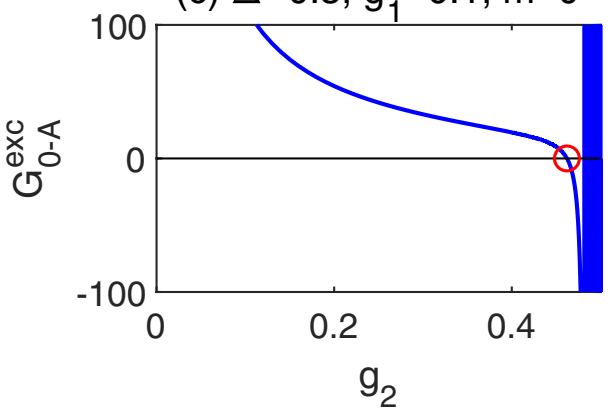

(b) $\Delta=0.5, g_{1}=0.1, m=1$

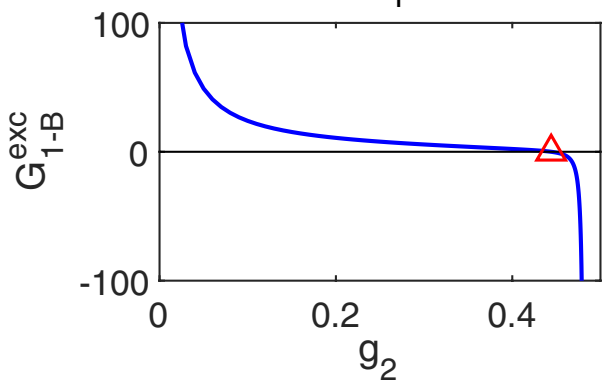

(d) $\Delta=0.5, g_{1}=0.1, m=1$

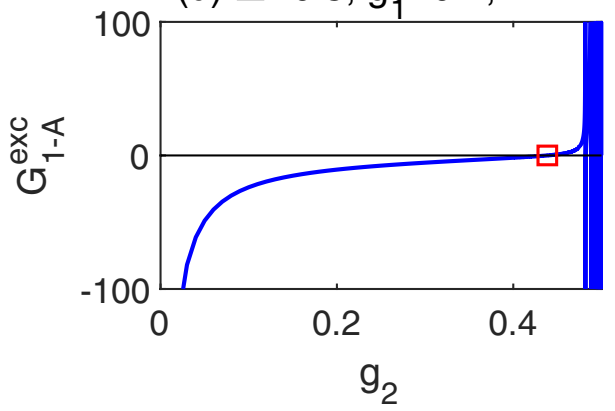

Figure 3. (a) Enlarged view of energy spectra in Fig. 2(a). The solid black lines denote the energy spectra obtained from the $G$-function. The blue dash (red dot) lines denote poles associated with $A(B)$ operator. The triangle denotes the first intersecting point of the energy levels and the 1-st pole line associated with the $B$ operator. The circle (square) denotes the intersecting point of the energy level and the 0 -th (1-st) pole line associated with the $A$ operator. (b) The B-type exceptional eigenvalue for $m=1$. The A-type exceptional eigenvalues for (c) $m=0$ and (d) $m=1 . \Delta=0.5, g_{1}=0.1$.

Pole structure and collapse. From Eqs. (13) and (16), we can find two kinds of poles associated with the $A$ and $B$ operators respectively, which will lead to the divergency of the recurrence relations,

$$
\begin{aligned}
& E_{n}^{\text {(pole_A })}=\beta n-\frac{1-\beta}{2}-\frac{g_{1}^{2}}{1+2 g_{2}}, \\
& E_{n}^{\text {(pole_B })}=\beta n-\frac{1-\beta}{2}-\frac{g_{1}^{2}}{1-2 g_{2}} .
\end{aligned}
$$

With the same $n$, the difference of two poles is independent of $n$.

$$
\Delta E^{(\mathrm{p})}=\frac{g_{1}^{2}}{1-2 g_{2}}-\frac{g_{1}^{2}}{1+2 g_{2}}=g_{1}^{2} \frac{4 g_{2}}{\beta^{2}} .
$$

In the limit of $g_{2} \rightarrow 1 / 2, \beta \rightarrow 0$, all $E_{n}$ (pole_A) are squeezed into a single finite value $-\frac{1}{2}\left(1+g_{1}^{2}\right)$, while all $E_{n}$ (pole_B) diverge to $-\infty$. It seems that there are two kinds of collapse energies. But actually, all energy levels tend to $B$-poles only, namely $-\infty$, if $g_{2} \rightarrow 1 / 2$, as shown in Fig. 2 . This spectral characteristics is quite different from the two-photon QRM where the energy spectra collapse to the finite value $e^{9,30,56}$. For the mixed QRM, the divergence of the eigenenergies to negative infinity for $g_{2} \rightarrow 1 / 2$ suggests some underlying unphysicality, which deserves further studies. The energies of the high excited states cross the pole A curves and then asymptotically converge to the pole $\mathrm{B}$, which lead to exceptional solutions.

Exceptional solutions. As shown in Fig. 3(a), most energy level curves pass through the pole curves on the way to $g_{2}=1 / 2$, which results in so-called exceptional solutions. They can be located in the following way.

At the intersecting point of the energy levels and the $m$-th pole line associated with the $A$-operator (26), the coefficient $f_{m}$ must vanish so that the pole is lift. Otherwise, the coefficient $e_{m}$ would diverge due to zero denominator in Eq. (13). In the unmixed QRM, $f_{m}=0$ can uniquely yield the necessary and sufficient condition for the occurrence of the exceptional solution. But here it is not that case, because $f_{m}$ depends on two initial variable $f_{0}, f_{1}$, and cannot be determined uniquely. The corresponding coefficient $e_{m}$ should be finite and can be regarded as an 
unknown variable. In all summations in Eqs. (20)-(23), the $m$-th terms should be treated specially, i.e. let $f_{m}$ be 0 and $e_{m}$ be a new variable. By the recurrence relation (14), we can add a new equation for this case

$$
f_{m}=\frac{-\frac{\Delta}{2} e_{m-2}+\left[\Omega(m-2, E)+h_{A}\right] f_{m-2}-2(u-v)^{2} w\left(f_{m-3}+(m-1) f_{m-1}\right)-2 u v f_{m-4}}{2 u v m(m-1)}=0 .
$$

So for the exceptional solution, we have a set of linear homogeneous equations (Eqs. (20-23) and (28)) with 5 unknown variables $f_{0}, f_{1}, f^{\prime}{ }_{0}, f^{\prime}{ }_{1}$ and $e_{m}$ for $m \geq 2$. While for $0 \leq m<2, f_{m}=0$, we have only 4 unknown variables $f_{1-m}, f^{\prime}{ }_{0}, f^{\prime}{ }_{1}$ and $e_{m}$, which can be determined by solving another set of linear homogeneous equations (Eqs. $(20-23))$. Nonzero solution requires the vanishing of the $5 \times 5(4 \times 4)$ determinant whose elements are just coefficients before $f_{0}, f_{1}, f^{\prime}{ }_{0}, f^{\prime}{ }_{1}$ and $e_{m}$ in Eqs. (20-23) and (28) $\left(f_{1-m}, f^{\prime}{ }_{0}, f^{\prime}{ }_{1}\right.$ and $e_{m}$ in Eqs. (20-23)) for $m \geq 2$ $(0 \leq m<2)$

$$
G_{m-A}^{\mathrm{exc}}\left(\Delta, g_{1}, g_{2}\right)=0 .
$$

We call this function as exceptional $G$-function. Here the energy is not an explicit variable, but determined by Eq. (26). The $m$-th exceptional solution associated with the $B$ operator can be detected in the same way by zero of an exceptional $G$-function

$$
G_{m-B}^{\mathrm{exc}}\left(\Delta, g_{1}, g_{2}\right)=0 .
$$

With the help of the exceptional $G$-function, we can determine the intersecting points of the energy levels and the pole curves as shown in Fig. 3(a) for $\Delta=0.5, g_{1}=0.1$. The $G^{\text {exc }}$ curves associated with $A$-pole as a function of $g_{2}$ for $m=0$ and 1 are shown in Fig. 3(c,d) respectively, and the $G^{\text {exc }}$ curve associated with $B$-pole for $m=1$ is shown in Fig. 3(b). The detected exceptional solutions in the $G^{\text {exc }}$ curves are marked by the same symbols as those in the enlarged spectra graph. One can find many zeros for $G^{\text {exc }}$ curve associated with $A$-pole in Fig. 3(c,d), which are corresponding to intersecting points of energy levels and the $m$-th $A$-pole curves as displayed in Fig. 3(a). For $G^{\text {exc }}$ curve associated with $B$-pole, there is only one zero for $m=1$ as exhibited in Fig. 3(b), also consistent with the single intersecting point shown in Fig. 3(a). No exceptional solution exists even for $B$-pole with $m=0$. All $g_{2}$ obtained from $G^{\text {exc }}=0$ can find their corresponding intersecting points in the energy spectra exactly.

Now we can judge whether it is the true level crossing or avoided crossing in the Fig. $2(\mathrm{a}-\mathrm{c})$. Around this regime, we have not found any exceptional solutions, indicating that the energy level cannot intersect with the pole curves. So although two energy levels are very close but blocked off by two pole curves with difference $\Delta E^{(\mathrm{p})} \propto g_{1}{ }^{2}$, they neither collide nor cross with each other. It is actually avoided crossing. For small $g_{1}, \Delta E^{(\mathrm{p})}$ is very small, so it looks like a "level crossing" as depicted in Fig. 2(a-c). For large $g_{1}$, the avoided crossing is quite clear, as shown in Fig. 2(d-f). Actually, these avoided crossings are just remnants of the traces of the doubly degeneracy in the unmixed model, which is relieved in the mixed model.

Effect of the mixed couplings. In the mixed QRM, if we combine Eqs. (5) and (8), the Hamiltonian (Eq. (2)) can be written as

$$
H=\left(\begin{array}{ll}
\frac{A^{\dagger} A-v^{2}-w^{2}}{u^{2}+v^{2}} & -\frac{\Delta}{2} \\
-\frac{\Delta}{2} & \frac{B^{\dagger} B-v^{2}-w^{\prime 2}}{u^{2}+v^{2}}
\end{array}\right),
$$

which can be reorganized and separated into three terms:

$$
H=H_{0}+\frac{\varepsilon^{(\mathrm{eff})}}{2} \sigma_{z}-\frac{1-\beta}{2} \mathbf{I}
$$

where $I$ is a unit matrix, and

$$
\begin{gathered}
H_{0}=\left(\begin{array}{cc}
\beta A^{\dagger} A-\frac{g_{1}^{2}}{\beta^{2}} & -\frac{\Delta}{2} \\
-\frac{\Delta}{2} & \beta B^{\dagger} B-\frac{g_{1}^{2}}{\beta^{2}}
\end{array}\right), \\
\varepsilon^{(\mathrm{eff})}=\frac{4 g_{2}}{1-4 g_{2}^{2}} g_{1}^{2} .
\end{gathered}
$$

An effective bias $\varepsilon^{\text {(eff) }}$ appears naturally, as well as a total energy shift $(1-\beta) / 2$. Recalling the one-photon $\mathrm{QRM}^{20}$,

$$
H_{1 \mathrm{P}}=-\frac{\Delta}{2} \sigma_{x}+\omega a^{\dagger} a+g_{1} \sigma_{z}\left(a^{\dagger}+a\right)
$$


this Hamiltonian can be expressed with a new set of bosonic operators $P=D^{\dagger}\left(g_{1}\right) a D\left(g_{1}\right)=a+g_{1}$ and $Q=D\left(g_{1}\right) a D^{\dagger}\left(g_{1}\right)=a-g_{1}$ as

$$
H_{1 \mathrm{P}}=\left(\begin{array}{ll}
\omega P^{\dagger} P-\frac{g_{1}^{2}}{\omega} & -\frac{\Delta}{2} \\
-\frac{\Delta}{2} & \omega Q^{\dagger} Q-\frac{g_{1}^{2}}{\omega}
\end{array}\right) .
$$

Comparing Eqs. (33) and (36), we can introduce an effective photon frequency $\omega^{\text {(eff) }}$ and an effective

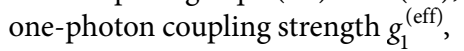

$$
\begin{gathered}
\omega^{(\mathrm{eff})}=\beta, \\
g_{1}^{(\mathrm{eff})}=\frac{g_{1}}{\sqrt{\beta}},
\end{gathered}
$$

and rewrite $H_{0}$ as

$$
H_{0}=-\frac{\Delta}{2} \sigma_{x}+\omega^{(\mathrm{eff})} a^{\dagger} a+g_{1}^{(\mathrm{eff})} \sigma_{z}\left(a^{\dagger}+a\right) .
$$

Therefore, we construct an effective one-photon QRM to describe the mixed one, which provides a more intuitional description of the influence of the mixed coupling,

$$
\begin{aligned}
H^{(\mathrm{eff})}= & \frac{\varepsilon^{(\mathrm{eff})}}{2} \sigma_{z}-\frac{\Delta}{2} \sigma_{x}-\frac{1-\beta}{2} \\
& +\omega^{(\mathrm{eff})} a^{\dagger} a+g_{1}^{(\mathrm{eff})} \sigma_{z}\left(a^{\dagger}+a\right) .
\end{aligned}
$$

Comparing $P(Q)$ with $A(B)$, the mainly difference is the lack of squeezing operator $S(r)$. The two-photon interaction leads to the squeezed field state $\mathrm{e}^{10,47}$, which can be well captured by the squeezing operator ${ }^{20,30,34}$. Therefore, the squeezing operator is introduced explicitly to deal with the mixed QRM, but it is not necessary for the one-photon model. The definitions of $P(Q)$ in Eq. (33) is equivalent with that of $A(B)$ in Eq. (36) only if $g_{2}=0$, so it is hard to use the effective Hamiltonian to deal with the strong two-photon coupling regime, especially the bosonic part due to the intense squeezing effect. We calculate the Wigner function $W\left(\alpha, \alpha^{*}\right)$ of the ground state ${ }^{57,58}$ in Fig. 4, which describes the probability distribution of the bosonic field in the phase space. When $g_{2}=0.1$, the differences of the Wigner functions calculated from $H$ and $H^{(\text {eff })}$ are negligible. However, It is shown in Fig. 4(c) that the squeezing effect becomes apparent for $H$ when $g_{2}=0.3$. The effective Hamiltonian can hardly describe the squeezing effect as demonstrated in Fig. 4(d). Nevertheless, it shed light on the analysis of strong coupling case, especially the properties of the two-level system. The ground-state magnetization $M=\left\langle\psi_{G S}\left|\sigma_{z}\right| \psi_{G S}\right\rangle$ calculated from $H^{(\text {eff) }}$ is in good agreement with that calculated from $H$ even in strong two-photon coupling regime, as shown in Fig. 5. When $g_{2}$ tends to $1 / 2$, the effective bias $\varepsilon^{\text {(eff) }}$ will tend to infinity, and the two-level system will prefer to stay in the lower level as indicated by the ground-state magnetization $M \rightarrow-1$ in Fig. 5 . Therefore, the energy contributed by $\varepsilon^{\text {(eff) }} \sigma_{z} / 2$ would be negative infinite, which is one of the reason for the negative divergence of the eigenenergies in the mixed QRM in the limit of $g_{2} \rightarrow 1 / 2$, as observed in Fig. 2. What is more, with the increase of $g_{2}$, the effective photon frequency $\omega^{\text {(eff) }}$ will decrease while the effective coupling $g_{1}^{\text {(eff) }}$ will increase, which might provide a novel and economic way to reach deep-strong one-photon coupling regime.

To further demonstrate the accuracy of the effective Hamiltonian, we calculate the dynamics of fidelity as shown in Fig. 6. The fidelity is defined as the overlap of the wavefunctions $\left|\psi^{(\text {eff })}(t)\right\rangle$ obtained from $H^{\text {(eff) }}$ (Eq. (37)) and $|\psi(t)\rangle$ obtained from $H$ (Eq. (1)), namely $F^{(\text {eff })}(t)=\left|\left\langle\psi^{(\text {eff })}(t) \mid \psi(t)\right\rangle\right|$, which can be used to judge how accurately the state of the effective Hamiltonian reproduces that of the original Hamiltonian. The initial states are $|\uparrow\rangle|0\rangle$ for both of them. The fidelity of the unbiased one-photon QRM, namely $H_{1 \mathrm{P}}$ (Eq. (35)), is also presented for comparison, i. e. $F_{1 P}(t)=\left|\left\langle\psi_{1 P}(t) \mid \psi(t)\right\rangle\right|$. When $g_{2}$ is as small as 0.05 , the corresponding effective bias reaches $\varepsilon^{(\text {eff })} \simeq 0.202$. This effective bias is large enough to play a significant role in the evolution of fidelity, as clearly seen in the Fig. 6(a). The fidelity of $H^{\text {(eff) }}$ tends to one, which is a strong evidence of the equivalence between $\left|\psi^{\text {(eff) }}(t)\right\rangle$ and $|\psi(t)\rangle$. The fidelity of $H_{1 \mathrm{P}}$ is much smaller, indicating that it deviates from the original Hamiltonian significantly. When we further increase $g_{2}$, the effective Hamiltonian still gives considerably good results while the deviation of $H_{1 \mathrm{p}}$ becomes more obvious. The fidelity of $H^{\text {(eff) }}$ drops slightly in the long time, which is mainly due to the error accumulation. The results of fidelity confirm the limitation of the effective Hamiltonian in dealing with the strong two-photon coupling and long-time limits.

The mixed QRM can be realized in the experiment by coupling the flux qubit to the plasma mode of its DC-SQUID detector ${ }^{11}$. We expect that the effective Hamiltonian can be employed to explain the experimental results. One of the most widely measured quantity in experiments is the transmission spectrum ${ }^{3,59,60}$. The transmission spectrum, i.e. $\delta E_{n}=E_{n}-E_{0}$, from both original full model (1) and the effective model (37) are shown in Fig. 7. We introduce an additional bias $\varepsilon \sigma_{z} / 2$ which is originated from the an externally applied magnetic flux in circuit QED system, and Eqs. (1) and (37) become 

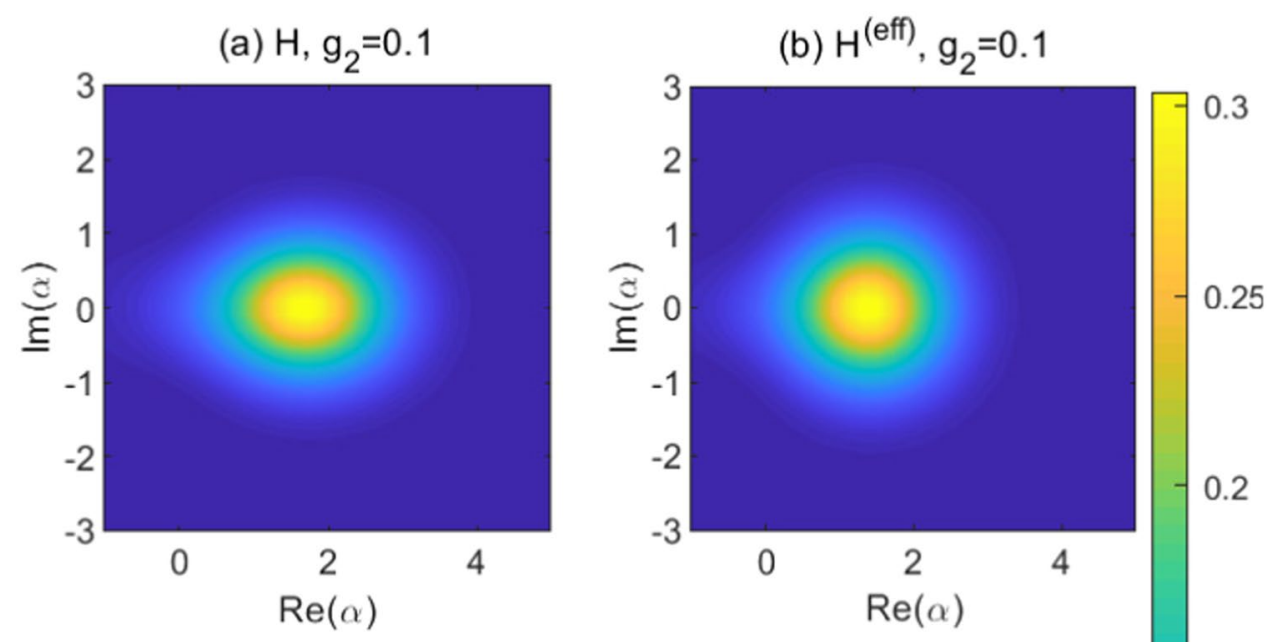

0.2
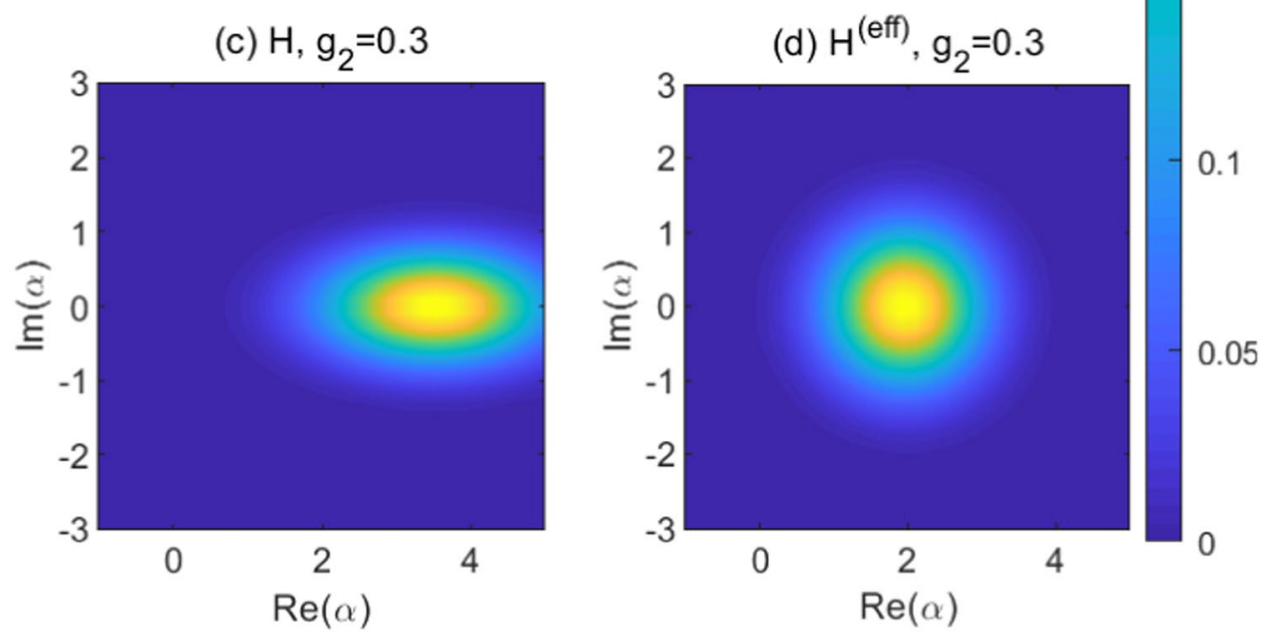

Figure 4. The Wigner function of the ground state at $\Delta=1, g_{1}=1$, with (a,b) $g_{2}=0.1$ and (c,d) $g_{2}=0.3$. Left column is calculated from the original Hamiltonian $H$, and the right column is calculated from the effective Hamiltonian $H^{(\text {eff })}$.

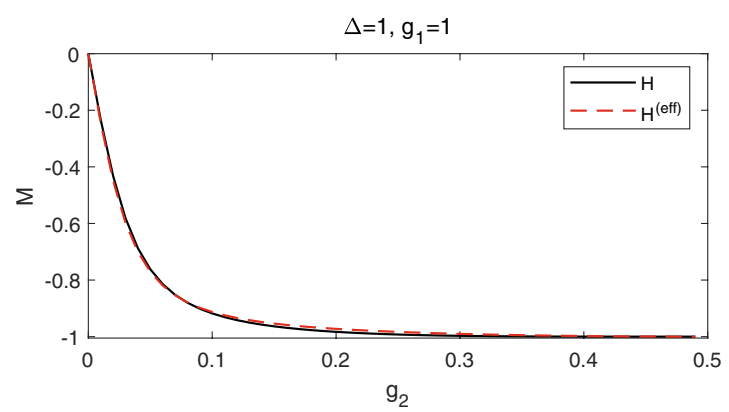

Figure 5. The ground-state magnetization $M$ as a function of $g_{2}$ for $\Delta=1, g_{1}=1$. Results for the full mixed model (1) are denoted by black solid lines, agreeing well with those by effective Hamiltonian (37) denoted by red dashed lines.

$$
\begin{aligned}
H_{\varepsilon}= & \frac{\varepsilon}{2} \sigma_{z}-\frac{\Delta}{2} \sigma_{x}+a^{\dagger} a \\
& +\sigma_{z}\left(g_{1}\left(a^{\dagger}+a\right)+g_{2}\left[\left(a^{\dagger}\right)^{2}+a^{2}\right]\right),
\end{aligned}
$$




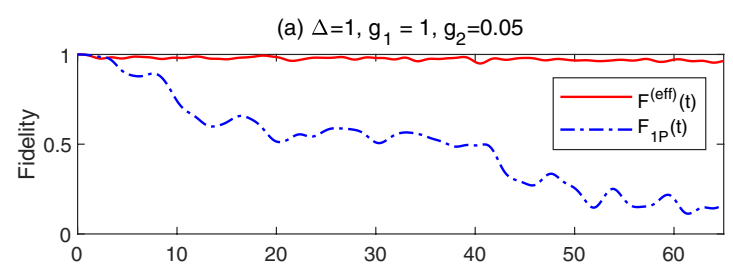

(b) $\Delta=1, g_{1}=1, g_{2}=0.1$

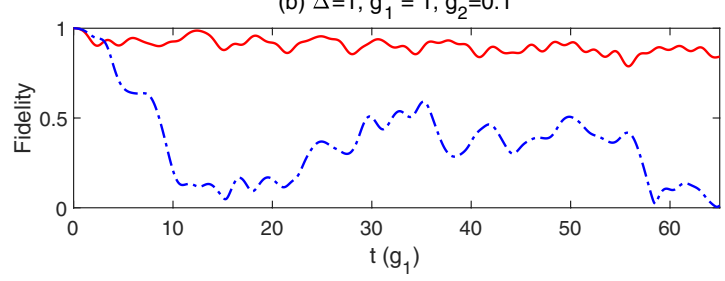

Figure 6. The fidelity of $H^{\text {(eff) }}$ (red sold line) and $H_{1 \mathrm{P}}$ (blue dash-dot line) as a function of $t$ for $\Delta=1, g_{1}=1,(\mathbf{a})$ $g_{2}=0.05$ and $(\mathbf{b}) g_{2}=0.1$.

(a) $\Delta=0.5, g_{1}=1, g_{2}=0.1$

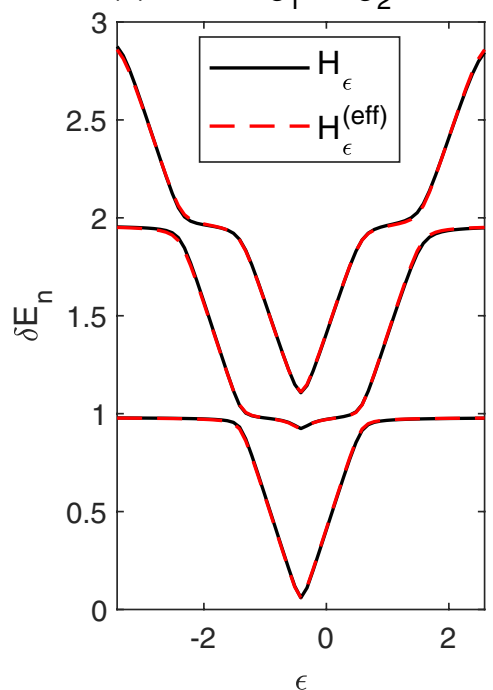

(b) $\Delta=0.5, g_{1}=1, g_{2}=0.45$

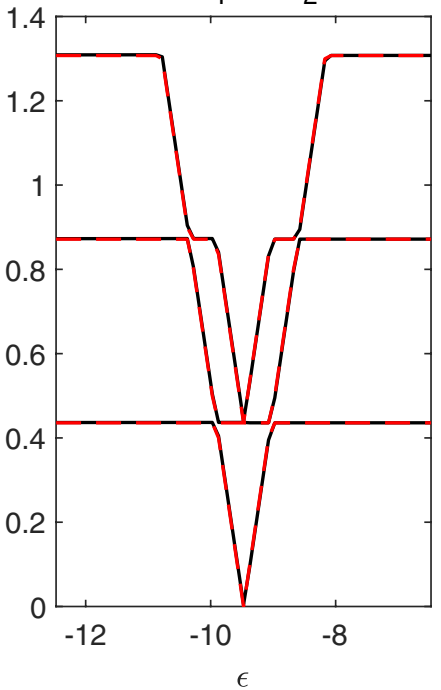

(c) $\Delta=0.5, g_{1}=0.3, g_{2}=0.45$

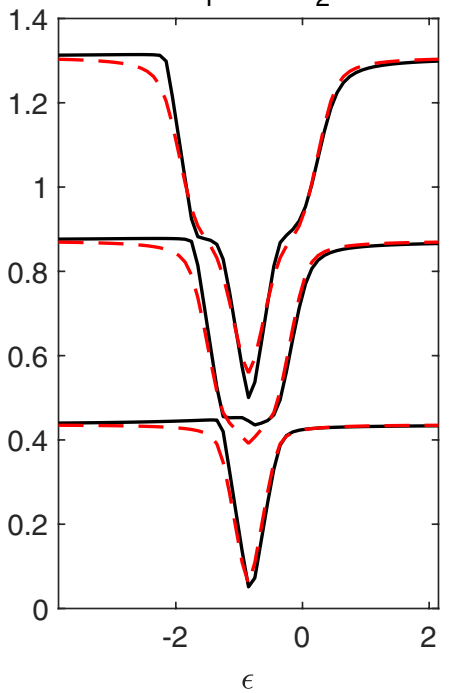

Figure 7. The energy difference $\delta E_{n}$ as a function of $\varepsilon$ for (a) $\Delta=0.5, g_{1}=1, g_{2}=0.1$, (b) $\Delta=0.5, g_{1}=1$, $g_{2}=0.45$, and (c) $\Delta=0.5, g_{1}=0.3, g_{2}=0.45$. Results calculated from $H_{\varepsilon}$, and $H_{\varepsilon}{ }^{\text {(eff) }}$ are marked with black solid and red dash lines respectively.

$$
\begin{aligned}
H_{\varepsilon}^{(\mathrm{eff})}= & \frac{\varepsilon+\varepsilon^{(\mathrm{eff})}}{2} \sigma_{z}-\frac{\Delta}{2} \sigma_{x} \\
& +\omega^{(\mathrm{eff})} a^{\dagger} a+g_{1}^{(\mathrm{eff})} \sigma_{z}\left(a^{\dagger}+a\right) .
\end{aligned}
$$

Therefore, the total bias in the effective Hamiltonian is $\left(\varepsilon^{(\mathrm{eff})}+\varepsilon\right)$. It is obvious from Fig. $7(\mathrm{a})$ that the effective Hamiltonian can capture the effects of two-photon coupling in the weak coupling regime very well. Even for strong two-photon coupling $g_{2}=0.45$, the effective Hamiltonian (39) still provides quite accurate energy structure and almost captures all features of the mixed QRM, as shown in Fig. 7(b,c). With the decrease of $g_{1}$, the deviation appears gradually because the two-photon interaction becomes dominated. One should also note that the energy differences of the effective Hamiltonian (Eq. (39)) is symmetry about $\varepsilon=-\varepsilon^{(\text {eff) }}$, which is different from that of the original one (Eq. (38)), as shown in Fig. 7(c). For the effective Hamiltonian with an additional bias, we can easy confirm that only the absolute value of $\left(\varepsilon+\varepsilon^{(\text {eff })}\right)$ affects the eigenenergies, as well as the energy differences. For the mixed QRM, this symmetry is broken due to the two-photon interaction term. Therefore, the asymmetry in the transmission spectrum can be regarded as a signature of the mixed one- and two-photon couplings. Far from the symmetry point $\varepsilon=-\varepsilon^{(\text {eff })}$, the energy difference tends to be a multiple of the photon frequency. The energy difference decreases with the increase of $g_{2}$, which can be explained by the suppressed effective photon frequency $\omega^{(\text {eff })}$. 

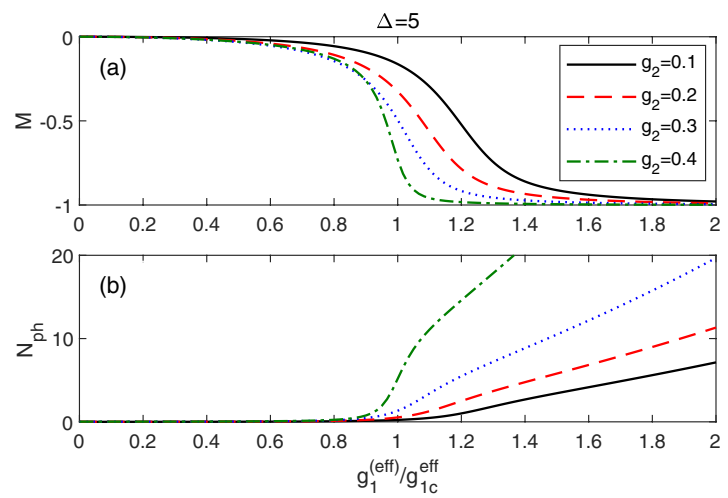

Figure 8. (a) The magnetization $M$ and (b) photon number $N_{\mathrm{ph}}$ calculated from $H$ as a function of $g_{1}^{\text {(eff) }}$ for $\Delta=5, g_{2}=0.1$ (black solid), 0.2 (red dash), 0.3 (blue dot) and 0.4 (green dash-dot lines).

Recently, the quantum phase transition of one-photon QRM in $\Delta / \omega \rightarrow \infty$ has drawn much attention ${ }^{48,49}$. The magnetization $M=\left\langle\sigma_{z}\right\rangle$ serves as an order parameter which changes from zero in the normal phase to nonzero in the superradiant phase when $g_{1}$ crosses the critical point $g_{1 c}=\sqrt{\Delta \omega} / 2$. Above $g_{1 c}$, photons are extremely activated as well. The ground-state magnetization $M$ and the photon number $N_{\mathrm{ph}}=\left\langle a^{\dagger} a\right\rangle$ calculated from $H$ (Eq. (1))

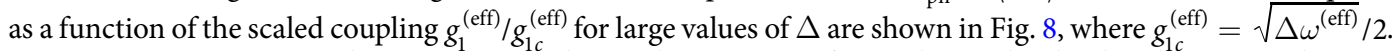
A negative $M$ emerges in the mixed QRM due to the positive effective bias. Clearly, the photon number $N_{\text {ph }}$ increases with the increase of $g_{2}$, which also indicates that the qubit-cavity interactions are enhanced and more photons are excited. For different two-photon coupling in the mixed QRM, the photons are considerably enhanced at almost the same scaled coupling around 1 . Whether it is a signature of the quantum phase transitions in the mixed QRM as observed in ref. ${ }^{50,61}$. deserves further study.

\section{Summary}

In this paper, by using Bogoliubov operators, we exactly solve the mixed QRM with both one- and two-photon terms analytically. The $G$-functions with the pole structures are derived, which reproduce completely the regular spectra. They can also be reduced to the unmixed ones. It is found that there are two sets of poles associated with two Bogoliubov operators. Two types of exceptional eigenvalues are then derived, which cannot be obtained solely by requiring that the corresponding coefficients vanish like in the unmixed models. When the two-photon coupling strength $g_{2}$ is close to $1 / 2$, two collapse energies are derived. One is finite, while the other diverges negatively. All energy levels collapse to the lower one, therefore diverge also, in sharp contrast to the unmixed two-photon model. The level degeneracy in the unmixed model is relieved due to the absence of parity symmetry. The avoided crossings are strictly discerned from the very close levels in the mixed model by the absence of exceptional eigenvalues around the "crossings".

We construct an effective one-photon Hamiltonian to describe the the mixed QRM, which is valid in weak two-photon coupling regime. The mixed QRM is equivalent to a single-photon one with an effective positive bias, suppressed photon frequency and enhanced one-photon coupling. This feature in the mixed system is very helpful to the recent circuit QED experiments where the intense competition to increase one-photon coupling is performed in many groups $s^{2,3,59,60}$. We suggest that the simultaneous presence of both one- and two-photon couplings would cooperate to provide richer physics.

Received: 10 September 2019; Accepted: 13 November 2019;

Published online: 04 December 2019

\section{References}

1. Braak, D., Chen, Q. H., Batchelor, M. T. \& Solano, E. Semi-classical and quantum Rabi models: in celebration of 80 years. J. Phys. A 49, 300301 (2016).

2. Niemczyk, T. et al. Circuit quantum electrodynamics in the ultrastrong-coupling regime. Nat. Phys. 6, 772 (2010).

3. Forn-Daz, P. et al. Observation of the Bloch-Siegert shift in a qubit-oscillator system in the ultrastrong coupling regime. Phys. Rev. Lett. 105, 237001 (2010).

4. Leibfried, D., Blatt, R., Monroe, C. \& Wineland, D. Quantum dynamics of single trapped ions. Rev. Mod. Phys. 75, 281 (2003).

5. Pedernales, J. S. et al. Quantum Rabi model with trapped ions. Sci. Rep. 5, 15472 (2015).

6. Hennessy, K. et al. Quantum nature of a strongly coupled single quantum dot-cavity system. Nature. 445, 896 (2007).

7. Bertet, P. et al. Generating and probing a two-photon Fock state with a single atom in a cavity. Phys. Rev. Lett. 88, 143601 (2002).

8. Brune, M., Raimond, J. M., Goy, P., Davidovich, L. \& Haroche, S. Realization of a two-photon maser oscillator. Phys. Rev. Lett. 59, 1899 (1987).

9. Felicetti, S. et al. Spectral collapse via two-phonon interactions in trapped ions. Phys. Rev. A 92, 033817 (2015).

10. Felicetti, S., Rossatto, D. Z., Rico, E., Solano, E. \& Forn-Daz, P. Two-photon quantum Rabi model with superconducting circuits. Phys. Rev. A 97, 013851 (2018).

11. Bertet, P., Chiorescu, I., Harmans, C. J. P. \& Mooij, J. E. Dephasing of a flux-qubit coupled to a harmonic oscillator. Preprint at arXiv:cond-mat/0507290(2005).

12. Bertet, P. et al. Dephasing of a superconducting qubit induced by photon noise. Phys. Rev. Lett. 95, 257002 (2005).

13. Chen, Z. et al. Single-photon-driven high-order sideband transitions in an ultrastrongly coupled circuit-quantum-electrodynamics system. Phys. Rev. A 96, 012325 (2017). 
14. Casanova, J., Puebla, R., Moya-Cessa, H. \& Plenio, M. B. Connecting $n$th order generalised quantum Rabi models: Emergence of nonlinear spin-boson coupling via spin rotations. npj Quantum Information 4, 47 (2018).

15. Pedernales, J. S. Dirac equation in (1+1)-dimensional curved spacetime and the multiphoton quantum Rabi model. Phys. Rev. Lett. 120, $160403(2018)$

16. Xie, Q. T., Zhong, H. H., Batchelor, M. T. \& Lee, C. H. The quantum Rabi model: solution and dynamics. J. Phys. A 50, 113001 (2017).

17. Gu, X., Kockum, A. F., Miranowicz, A., Liu, Y. X. \& Nori, F. Microwave photonics with superconducting quantum circuits. Physics Reports 718, 1-102 (2017).

18. Forn-Daz, P., Lamata, L., Rico, E., Kono, J. \& Solano, E. Ultrastrong coupling regimes of light-matter interaction. Rev. Mod. Phys. 91, 025005 (2019).

19. Braak, D. Integrability of the Rabi model. Phys. Rev. Lett. 107, 100401 (2011).

20. Chen, Q. H., Wang, C., He, S., Liu, T. \& Wang, K. L. Exact solvability of the quantum Rabi model using Bogoliubov operators. Phys. Rev. A 86, 023822 (2012).

21. Zhong, H., Xie, Q., Batchelor, M. T. \& Lee, C. Analytical eigenstates for the quantum Rabi model. J. Phys. A 46, 415302 (2013).

22. Maciejewski, A. J., Przybylska, M. \& Stachowiak, T. Analytical method of spectra calculations in the Bargmann representation. Phys. Lett. A 378, 3445 (2014).

23. Chilingaryan, S. A. \& Rodríguez-Lara, B. M. The quantum Rabi model for two qubits. J. Phys. A 46, 335301 (2013).

24. Peng, J. et al. Solution of the two-qubit quantum Rabi model and its exceptional eigenstates. J. Phys. A 47, 265303 (2014).

25. Wang, H., He, S., Duan, L., Zhao, Y. \& Chen, Q. H. Solutions to the quantum Rabi model with two equivalent qubits. EPL 106, 54001 (2014).

26. Xie, Q. T., Cui, S., Cao, J. P., Amico, L. \& Fan, H. Anisotropic rabi model. Phys. Rev. X 4, 021046 (2014).

27. Travěnec, I. Solvability of the two-photon Rabi Hamiltonian. Phys. Rev. A 85, 043805 (2012).

28. Maciejewski, A. J., Przybylska, M. \& Stachowiak, T. Comment on "Solvability of the two-photon Rabi Hamiltonian". Phys. Rev. A 91, 037801 (2015).

29. Travěnec, I. Reply to "Comment on'Solvability of the two-photon Rabi Hamiltonian'. Phys. Rev. A 91, 037802 (2015).

30. Duan, L., Xie, Y. F., Braak, D. \& Chen, Q. H. Two-photon Rabi model: analytic solutions and spectral collapse. J. Phys. A 49, 464002 (2016).

31. Zhang, Y. Z. On the solvability of the quantum Rabi model and its 2-photon and two-mode generalizations. J. Math. Phys. 54, 102104 (2013).

32. Zhang, Y. Z. Hidden sl (2)-algebraic structure in Rabi model and its 2-photon and two-mode generalizations. Ann. Phys 375, 460 (2016).

33. Maciejewski, A. J. \& Stachowiak, T. A novel approach to the spectral problem in the two photon Rabi model. J. Phys. A 50, 244003 (2017).

34. Lupo, E., Napoli, A., Messina, A., Solano, E. \& Egusquiza, I. L. A continued fraction based approach for the Two-photon Quantum Rabi Model. Sci. Reps. 9, 4156 (2019).

35. Feranchuk, I. D., Komarov, L. I. \& Ulyanenkov, A. P. Two-level system in a one-mode quantum field: numerical solution on the basis of the operator method. J. Phys. A 29, 4035 (1996).

36. Irish, E. K. Generalized rotating-wave approximation for arbitrarily large coupling. Phys. Rev. Lett. 99, 173601 (2007).

37. Chen, Q. H., Zhang, Y. Y., Liu, T. \& Wang, K. L. Numerically exact solution to the finite-size Dicke model. Phys. Rev. A 78, 051801(R) (2008).

38. Gan, C. J. \& Zheng, H. Dynamics of a two-level system coupled to a quantum oscillator: transformed rotating-wave approximation. Eur. Phys. J. D 59, 473 (2010).

39. He, S. et al. First-order corrections to the rotating-wave approximation in the Jaynes-Cummings model. Phys. Rev. A 86, 033837 (2012).

40. He, S., Zhao, Y. \& Chen, Q. H. Absence of collapse in quantum Rabi oscillations. Phys. Rev. A 90, 053848 (2014).

41. Mao, L., Huai, S. \& Zhang, Y. The two-qubit quantum Rabi model: inhomogeneous coupling. J. Phys. A 48, 345302 (2015).

42. Ying, Z. J., Liu, M., Luo, H. G., Lin, H. Q. \& You, J. Q. Ground-state phase diagram of the quantum Rabi model. Phys. Rev. A 92, 053823 (2015).

43. Zhang, Y. Y. Generalized squeezing rotating-wave approximation to the isotropic and anisotropic Rabi model in the ultrastrongcoupling regime. Phys. Rev. A 94, 063824 (2016).

44. Lü, Z., Zhao, C. \& Zheng, H. Quantum dynamics of two-photon quantum Rabi model. J. Phys. A 50, 074002 (2017).

45. Cong, L., Sun, X. M., Liu, M., Ying, Z. J. \& Luo, H. G. Polaron picture of the two-photon quantum Rabi model. Phys. Rev. A 99, $013815(2019)$

46. Casanova, J., Romero, G., Lizuain, I., Garca-Ripoll, J. J. \& Solano, E. Deep strong coupling regime of the Jaynes-Cummings model. Phys. Rev. Lett. 105, 263603 (2010).

47. Peng, J. S. \& Li, G. X. Influence of the virtual-photon processes on the squeezing of light in the two-photon Jaynes-Cummings model. textitPhys. Rev. A 47, 3167 (1993).

48. Hwang, M. J., Puebla, R. \& Plenio, M. B. Quantum phase transition and universal dynamics in the Rabi model. Phys. Rev. Lett. 115, 180404 (2015).

49. Liu, M. X. et al. Universal scaling and critical exponents of the anisotropic quantum Rabi model. Phys. Rev. Lett. 119, 220601 (2017).

50. Peng, J., Rico, E., Zhong, J., Solano, E. \& Egusquiza, I. L. Unified Superradiant phase transitions. Preprint at arXiv:1904.02118 (2019).

51. Malekakhlagh, M. \& Rodriguez, A. W. Quantum Rabi model with two-photon relaxation. Phys. Rev. Lett. 122, 043601 (2019).

52. Xie, Y. F., Duan, L. \& Chen, Q. H. Generalized quantum Rabi model with both one-and two-photon terms: A concise analytical study. Phys. Rev. A 99, 013809 (2019).

53. Duan, L., He, S., Braak, D. \& Chen, Q. H. Solution of the two-mode quantum Rabi model using extended squeezed states. EPL 112, 34003 (2015).

54. Braak, D. Solution of the Dicke model for $\mathrm{N}=3$. J. Phys. B 46, 224007 (2013).

55. He, S., Duan, L. \& Chen, Q. H. Exact solvability, non-integrability, and genuine multipartite entanglement dynamics of the Dicke model. New J. Phys. 17, 043033 (2015).

56. Ng, K. M., Lo, C. F. \& Liu, K. L. Exact eigenstates of the two-photon Jaynes-Cummings model with the counter-rotating term. Eur. Phys. J. D 6, 119 (1999).

57. Johansson, J. R., Nation, P. D. \& Nori, F. QuTiP 2: A Python framework for the dynamics of open quantum systems. textitComp. Phys. Comm. 184, 1234 (2013).

58. Johansson, J. R., Nation, P. D. \& Nori, F. QuTiP: An open-source Python framework for the dynamics of open quantum systems. textitComp. Phys. Comm. 183, 1760 (2012).

59. Yoshihara, F. et al. Superconducting qubit-oscillator circuit beyond the ultrastrong-coupling regime. Nat. Phys. 13, 44 (2017).

60. Forn-Daz, P. et al. Ultrastrong coupling of a single artificial atom to an electromagnetic continuum in the nonperturbative regime. Nat. Phys. 13, 39 (2017).

61. Ying, Z. J., Cong, L. \& Sun, X. M. Quantum phase transition and spontaneous symmetry breaking in a nonlinear quantum Rabi model. Preprint at arXiv:1804.08128 (2018). 


\section{Acknowledgements}

This work is supported by the National Key Research and Development Program of China (No. 2017YFA0303002), the National Science Foundation of China (Grants No. 11674285 and No. 11834005).

\section{Author contributions}

Q.-H. Chen conceived the idea and contributed to the theoretical analysis and interpretation of data, and wrote the manuscript. L. Duan performed the numerical calculations and contributed to the interpretation of the numerical results, and contributed to the writing of the manuscript. Y.-F. Xie contributed to the interpretation of the numerical results. All authors reviewed the manuscript.

\section{Competing interests}

The authors declare no competing interests.

\section{Additional information}

Correspondence and requests for materials should be addressed to Q.-H.C.

Reprints and permissions information is available at www.nature.com/reprints.

Publisher's note Springer Nature remains neutral with regard to jurisdictional claims in published maps and institutional affiliations.

Open Access This article is licensed under a Creative Commons Attribution 4.0 International License, which permits use, sharing, adaptation, distribution and reproduction in any medium or format, as long as you give appropriate credit to the original author(s) and the source, provide a link to the Creative Commons license, and indicate if changes were made. The images or other third party material in this article are included in the article's Creative Commons license, unless indicated otherwise in a credit line to the material. If material is not included in the article's Creative Commons license and your intended use is not permitted by statutory regulation or exceeds the permitted use, you will need to obtain permission directly from the copyright holder. To view a copy of this license, visit http://creativecommons.org/licenses/by/4.0/.

(c) The Author(s) 2019 